DESY 99-084

ISSN 0418-9833

MZ-TH/99-25

TTP99-32

hep-ph/9907511

July 1999

\title{
Photon Fragmentation in Large- $Q^{2}$ ep Collisions at Next-to-Leading Order QCD
}

\author{
A. Gehrmann-De Ridder ${ }^{1}$, G. Kramer ${ }^{2}$ and H. Spiesberger ${ }^{3}$ \\ ${ }^{1}$ Deutsches Elektronen-Synchrotron DESY, D-22603 Hamburg, Germany, and \\ Institut für Theoretische Teilchenphysik, Universität Karlsruhe, \\ D-76128 Karlsruhe, Germany \\ 2 II. Institut für Theoretische Physik, Universität Hamburg, \\ Luruper Chaussee 149, D-22761 Hamburg, Germany \\ ${ }^{3}$ Institut für Physik, Johannes-Gutenberg-Universität, \\ Staudinger Weg 7, D-55099 Mainz, Germany \\ e-mail: gehra@particle.physik.uni-karlsruhe.de, \\ kramer@mail.desy.de, hspiesb@thep.physik.uni-mainz.de
}

\begin{abstract}
We study the production of photons accompanied by jets in large- $Q^{2}$ deep inelastic scattering. Numerical results for the cross section differential with respect to the fraction of momentum $z_{\gamma}$ carried by a photon inside a jet at large $z_{\gamma}$, up to $O\left(\alpha \alpha_{s}\right)$ in perturbative QCD, are presented. The sensitivity to the fragmentation contribution allows one to study the quark-to-photon fragmentation function. Our results can be confronted with future experimental data from HERA.
\end{abstract}

\footnotetext{
*Supported by Bundesministerium für Forschung und Technologie, Bonn, Germany, under Contract $057 \mathrm{HH}$ 92P (0), and by EU Fourth Framework Program Training and Mobility of Researchers through Network Quantum Chromodynamics and Deep Structure of Elementary Particles under Contract FMRXCT98-0194 (DG12 MIHT).
} 


\section{Introduction}

The production of final state photons at large transverse momenta in high-energy processes is an important observable for testing QCD. Data on large- $p_{T}$ photon production in hadronic collisions have been used in the past to obtain information on the gluon distribution in the photon. In addition, a good understanding of direct photon production within the framework of the standard model will be essential for new physics searches at future colliders.

Photons from high-energy collisions are produced essentially by two mechanisms: the direct production of a photon off a primary quark or antiquark, or the fragmentation of a hadronic jet into a single photon carrying a large fraction of the jet energy. The direct production gives rise to perturbatively calculable short-distance contributions whereas the fragmentation contribution is due primarily to a long distance process which can not be calculated completely inside perturbative QCD. The latter is described by the process-independent quark-, antiquark-, or gluon-to-photon fragmentation functions (FF) [1] which must be fixed by experimental data as long as they can not be calculated by nonperturbative methods. Their evolution with the factorization scale $\mu_{F}$ and their so-called point-like contribution (up to a normalization) can, however, be calculated perturbatively.

Directly produced photons are usually well separated from hadron jets, while photons originating from the fragmentation process are primarily found inside hadronic jets. By imposing an isolation criterion on the photon, one can, in principle, suppress (but in general, not eliminate) the fragmentation contribution to the final state photon cross section円.

So far, only a limited number of measurements of single photon production exists through which direct information on the photon FF can be obtained. A possible way is the measurement of the inclusive photon cross section in $e^{+} e^{-}$annihilation. Recently the OPAL collaboration at LEP has measured this cross section for final state photons in the range $0.2<x_{\gamma}<1.0$, where $x_{\gamma}=2 E_{\gamma} / M_{Z}$ is the photon energy fraction in terms of the beam energy [3]. The results are in reasonable agreement with predictions obtained within perturbative QCD using the model-dependent parametrizations of the photon FF of DO [4], GRV [5] or BFG [6], when choosing the factorization scale $\mu_{F}=M_{Z}$. The DO model for the $\mathrm{FF}$ is based on an asymptotic solution of the evolution equation whereas the other two models [5, 6] contain in addition a non-perturbative input inspired by the vector meson dominance model. Unfortunately, the experimental precision was not sufficiently high to discriminate between these theoretical predictions. Note also that in [7, 8] it was shown that predictions obtained within perturbative QCD and using a fixed-order expanded expression for the photon FF also agreed well with the OPAL data.

An alternative way to determine the $\mathrm{FF}$ from $e^{+} e^{-}$data is to measure the production of photons accompanied with a definite number of hadronic jets [10]. In this approach, the photon is treated like any other hadron and is clustered simultaneously with the other

\footnotetext{
${ }^{1}$ See [2] for a proposal to eliminate the dependence on the photon FF.
} 
hadrons into jets (so-called democratic clustering procedure) [11]. Then, one of the jets in the final state is considered as the photon jet if the fraction of the electromagnetic energy inside the jet is sufficiently large, i.e.

$$
z=\frac{E_{\gamma}}{E_{\gamma}+E_{h a d}}>z_{c u t}
$$

with $z_{\text {cut }}$ fixed by the experimental conditions. On this basis the ALEPH collaboration at LEP analysed events produced at the $Z$-resonance [12] which contained one hadron jet and a jet with a photon carrying at least $70 \%$ of the jet energy. This $\gamma+1$-jet rate was used to determine the quark-to-photon FF, which was calculated in leading order (LO), i.e. up to $\mathrm{O}(\alpha)$, in [11]. The calculation was extended to next-to-leading order (NLO), i.e. up to $\mathrm{O}\left(\alpha \alpha_{s}\right)$, in [7, 9] and a NLO photon-to-quark FF was obtained [7] by comparing with the ALEPH data. Due to the unfavorable signal-to-background ratio below $z=0.7$ for this particular observable, the $\gamma+1$-jet rate, the photon FF could be determined only in the range $0.7<z<1.0$. In [8, 13] also the predictions of GRV [5] and BFG [6] for the photon FF were compared to the ALEPH $\gamma+1$-jet data. It turned out that the BFG parametrization is in agreement with the measurements while the rate predicted with the help of the FF of GRV lies too high.

The photon FF is process independent as any other FF and can be used to predict the cross section for the production of single photons in other processes. This has been done recently for isolated photon production in deep-inelastic ep scattering (DIS) [14. In that work the emphasis was put on the prediction of cross sections in the HERA kinematic range without the necessity to introduce photon-parton separation cuts as used in earlier work [15]. This was achieved by including the divergent photon-quark (-antiquark) singular contributions and the bare quark-to-photon fragmentation contributions, leaving in the final result only a finite factorization scale dependent quark-to-photon FF contribution. In our previous work we adopted as the photon FF the lowest-order fit obtained from the photon+1-jet data of the ALEPH collaboration [12].

In the present work we study the influence of the fragmentation contribution on the cross section for the production of a photon plus jets in DIS and give predictions for $d \sigma / d z_{\gamma}$ as a function of $z_{\gamma}$ in the interval $z_{c u t}=0.5<z_{\gamma}<1.0$ (for the definition of $z_{\gamma}$ see below). For this purpose we calculate $d \sigma / d z_{\gamma}$ with the same cuts on the DIS variables as in [14] for essentially three different sets of photon FF's, the ALEPH sets in LO and NLO [12, 7], the leading-logarithmic (LL) and beyond-leading logarithmic (BLL) parametrizations of GRV [5] and the BLL parametrization of BFG [6]. The first set of photon FF is obtained within a fixed-order framework at a given order in $\alpha_{s}$. The two other sets were obtained after the leading and/or next-to-leading logarithms of the factorization scale $\mu_{F}$ were resummed. The essence of these two approaches has been described extensively in [8] and will be outlined below. 


\section{Leading and Next-to-Leading Order Cross Section}

In leading orderf (i.e., at $\mathrm{O}(\alpha)$ ), the cross section for the production of photon plus jets in DIS receives contributions from the quark (antiquark) subprocess $\gamma^{*} q \rightarrow \gamma q\left(\gamma^{*} \bar{q} \rightarrow \gamma \bar{q}\right)$. Together with the remnant jet from the proton this process gives rise to a $\gamma+(1+1)$ jet final state. In the virtual photon-proton center-of-mass system the hard $\gamma$ recoils against the hard jet back-to-back. Cuts on the usual DIS variables $x, y$ and $Q^{2}$ are applied to remove $\gamma$ production by incoming $\gamma^{*}$ 's of small virtuality. To produce $\gamma^{\text {'s }}$ of sufficiently high energy, an explicit cut on the total $\gamma^{*} p$ center-of-mass energy, $W$, is introduced. Both leptons and quarks emit photons. The leptonic radiation is suppressed by a cut on the photon emission angle with respect to the incoming electron in the same way as in the earlier work [14, 15]. In LO each parton is identified with a jet and the photon is automatically isolated from the quark jet by demanding a non-zero transverse momentum of the photon or jet in the $\gamma^{*} p$ center-of-mass frame. At this order there is no fragmentation contribution.

At next-to-leading order $\left(\mathrm{O}\left(\alpha \alpha_{s}\right)\right)$ we have subprocesses with an additional gluon, either in the final or in the initial state, i.e. the subprocesses $\gamma^{*} q \rightarrow \gamma q g$ (and similarly with $q$ replaced by $\bar{q}$ ) and $\gamma^{*} g \rightarrow \gamma q \bar{q}$, respectively. In addition, virtual corrections (one-loop diagrams at $\left.\mathrm{O}\left(\alpha \alpha_{s}\right)\right)$ to the LO processes have to be included. The processes $\gamma^{*} q \rightarrow \gamma q g$ and $\gamma^{*} g \rightarrow \gamma q \bar{q}$ contribute both to the $\gamma+(1+1)$-jets cross section and to the cross section for $\gamma+(2+1)$-jets. In the latter case each parton in the final state (including the photon) builds a jet on its own, whereas for $\gamma+(1+1)$-jets a pair of final state partons is combined into one jet. The recombination of two partons will be performed with the help of the cone algorithm. The exact prescriptions will be given later when we present our results. In the NLO calculation of the $\gamma+(1+1)$-jet cross section one encounters the well known infrared and collinear singularities. For the processes $\gamma^{*} q \rightarrow \gamma q g$ and $\gamma^{*} g \rightarrow \gamma q \bar{q}$ they appear in those phase space regions where two partons are degenerate to one parton, i.e. when one of the partons becomes soft or two partons become collinear to each other. These singularities cancel in the case of soft gluons or for collinear quarkgluon pairs against the singularities from the virtual corrections to the LO process or have to be factorized and absorbed into the renormalized parton distribution functions (PDF's) of the proton. To accomplish this cancellation, the singularities are isolated in an analytic calculation with the help of dimensional regularization and the phase space slicing method. The technical details of this procedure have been described in [14, 15] and need not to be repeated here.

The matrix elements $|M|^{2}$ for the processes $\gamma^{*} q \rightarrow \gamma q g$ and $\gamma^{*} g \rightarrow \gamma q \bar{q}$ have also photonic infrared and collinear singularities. The infrared singularity is outside the kinematical region we are interested in since the photon is required to be observed in the detector. In the numerical calculation of the cross section we impose this condition by demanding a minimal transverse momentum of the final state photon. This cut removes also collinear

\footnotetext{
${ }^{2}$ Here and in the following, we do not count the extra factor $\alpha$ from the $e-\gamma^{*}$ vertex.
} 
singularities due to initial state photon radiation. Final state collinear singularities are present and are treated with the phase space slicing method similar to the case of the quark-gluon collinear contribution. For this purpose the phase space slicing parameter $y_{0}^{\gamma}$ is introduced, which is chosen very small, so that the matrix element $|M|^{2}$ can be approximated by its singular part. The slicing of the phase space is done with the help of the scaled squared invariant masses, for example, with $y_{\gamma q}=\left(p_{q}+p_{\gamma}\right)^{2} / W^{2}$ for the subprocess $\gamma^{*} q \rightarrow \gamma q g$, where $p_{\gamma}$ and $p_{q}$ are the four-momenta of the outgoing photon and quark, respectively. In the gluon initiated subprocess one has two singular regions, which are controlled by the variables $y_{\gamma q}$ and $y_{\gamma \bar{q}}$, respectively. In the region $y_{\gamma q}>y_{0}^{\gamma}$ the cross section is evaluated numerically. The details for the calculation of the various contributions are described in [14].

The contribution to $|M|^{2}$ in the region $y_{\gamma q}<y_{0}^{\gamma}$ is collinearly divergent and is regulated by dimensional regularization. The divergent part is absorbed into the bare photon FF to yield the renormalized photon $\mathrm{FF}$ denoted by $D_{q \rightarrow \gamma}$. The additional fragmentation contribution to $|M|^{2}$ for the subprocess $\gamma^{*} q \rightarrow \gamma q g$ has the following form

$$
|M|_{\gamma^{*} q \rightarrow \gamma q g}^{2}=|M|_{\gamma^{*} q \rightarrow q g}^{2} \otimes D_{q \rightarrow \gamma}(z) .
$$

There is a similar expression for the subprocess $\gamma^{*} g \rightarrow \gamma q \bar{q}$. It is obvious from (2) that the fragmentation contribution is $\mathrm{O}\left(\alpha \alpha_{s}\right)$, the photon $\mathrm{FF} D_{q \rightarrow \gamma}(z)$ given by

$$
D_{q \rightarrow \gamma}(z)=D_{q \rightarrow \gamma}\left(z, \mu_{F}^{2}\right)+\frac{\alpha e_{q}^{2}}{2 \pi}\left(P_{q \gamma}^{(0)}(z) \ln \frac{z(1-z) y_{0}^{\gamma} W^{2}}{\mu_{F}^{2}}+z\right)
$$

being of $\mathrm{O}(\alpha) . D_{q \rightarrow \gamma}\left(z, \mu_{F}^{2}\right)$ in (国) stands for the non-perturbative $\mathrm{FF}$ of the transition $q \rightarrow \gamma$ at the factorization scale $\mu_{F}$, i.e. the scale at which the redefinition has been performed. This function will be given by one of the sets of photon FF mentioned above. The second term in (3), if substituted in (2), is the finite part resulting from adding the bare photon $\mathrm{FF}$ and the collinear photon-quark (-antiquark) contribution to the matrix element $|M|_{\gamma^{*} q \rightarrow \gamma q g}^{2}$ integrated in the region $y_{\gamma q}<y_{0}^{\gamma}$.

The $y_{0}^{\gamma}$ dependence in (3) is expected to cancel the dependence of the numerically evaluated $\gamma+(1+1)$-jet cross section restricted to the region $y_{\gamma q}>y_{0}^{\gamma}$ as studied in 15 . This means that $y_{0}^{\gamma}$ is only a technical cut separating divergent from finite contributions. This collinear photon-quark (-antiquark) contribution to the matrix element $|M|_{\gamma^{*} q \rightarrow \gamma q g}^{2}$ being calculated in the collinear approximation is valid only up to terms $\mathrm{O}\left(y_{0}^{\gamma}\right)$. Consequently the cut $y_{0}^{\gamma}$ must be chosen sufficiently small. The $y_{0}^{\gamma}$ independence must be checked by varying $y_{0}^{\gamma}$. Results of this test will be shown below. In (3), $P_{q \gamma}^{(0)}(z)$ is the LO quark-to-photon splitting function

$$
P_{q \gamma}^{(0)}(z)=\frac{1+(1-z)^{2}}{z}
$$

and $e_{q}$ is the electric charge of quark $q$. The variable $z$ denotes the fraction of the final photon energy in terms of the energy of the quark emitting the photon. If the photon is 
emitted from a final state quark with four-momentum $p_{q_{4}^{\prime}}=p_{q_{4}}+p_{\gamma}$, then $z$ is obtained from $p_{\gamma}=z p_{q_{4}^{\prime}}$. It can also be related to the invariants $y_{q_{3} \gamma}$ and $y_{q_{3} q_{4}}$ obtained from the four-momenta of the subprocess $\gamma^{*} q_{3} \rightarrow \gamma q_{4} g$ with the result

$$
z=\frac{y_{q_{3} \gamma}}{y_{q_{3} q_{4}^{\prime}}}=\frac{y_{q_{3} \gamma}}{y_{q_{3} q_{4}}+y_{q_{3} \gamma}} .
$$

According to (2) the fragmentation contribution to the subprocess $\gamma^{*} q_{3} \rightarrow \gamma q_{4} g$ is calculated from the convolution of the FF with the $\mathrm{O}\left(\alpha_{s}\right)$ matrix element $|M|^{2}$ of the process $\gamma^{*} q_{3} \rightarrow q_{4} g$, which is well known. It yields a term of $\mathrm{O}\left(\alpha \alpha_{s}\right)$ to the cross section for $\gamma+(1+1)$-jets. Similarly the fragmentation contribution to the subprocess $\gamma^{*} g \rightarrow \gamma q \bar{q}$ is calculated from the convolution of the FF for $q \rightarrow \gamma$ with the matrix element $|M|^{2}$ of the process $\gamma^{*} g \rightarrow q \bar{q}$ which is also known. Equivalent formulas are used for the calculation of the fragmentation contribution to $\gamma^{*} \overline{q_{3}} \rightarrow \gamma \overline{q_{4}} g$ and $\gamma^{*} g \rightarrow \gamma q \bar{q}$, where the $\bar{q}$ fragments into a photon. The fragmentation is always assumed to occur collinearly, i.e. with no additional transverse momentum in the fragmentation process.

\section{Quark-to-Photon Fragmentation Functions}

As long as we intend to calculate the $\gamma+(n+1)$-jet cross section only up to $\mathrm{O}\left(\alpha \alpha_{s}\right)$ we need the photon FF only in LO, i.e. at $\mathrm{O}(\alpha)$. This means that the gluon-to-photon FF, which starts at $\mathrm{O}\left(\alpha \alpha_{s}\right)$, is not needed. In LO the non-perturbative quark-to-photon FF obeys the evolution equation

$$
\frac{d D_{q \rightarrow \gamma}\left(z, \mu_{F}\right)}{d \ln \mu_{F}^{2}}=\frac{\alpha e_{q}^{2}}{2 \pi} P_{q \gamma}^{(0)}(z)
$$

with the solution

$$
D_{q \rightarrow \gamma}\left(z, \mu_{F}\right)=\frac{\alpha e_{q}^{2}}{2 \pi} P_{q \gamma}^{(0)}(z) \ln \left(\frac{\mu_{F}^{2}}{\mu_{0}^{2}}\right)+D_{q \rightarrow \gamma}\left(z, \mu_{0}\right) .
$$

$D_{q \rightarrow \gamma}\left(z, \mu_{0}\right)$ is an initial value which must be fitted to experimental data with a chosen initial scale $\mu_{0}$. This has been done by the ALEPH collaboration [12]. From the fit to their $e^{+} e^{-} \rightarrow \gamma+1$-jet data [12] they obtained

$$
D_{q \rightarrow \gamma}^{L O}\left(z, \mu_{0}\right)=\frac{\alpha e_{q}^{2}}{2 \pi}\left(-P_{q \gamma}^{(0)}(z) \ln (1-z)^{2}-13.26\right)
$$

with $\mu_{0}=0.14 \mathrm{GeV}$. (7) together with (8) is one of the photon FF choices which will be used to predict the cross section $d \sigma / d z_{\gamma}$ for $\gamma+(n+1)$-jet production in DIS ep scattering. We note that (7) is an exact solution of (6) at $\mathrm{O}(\alpha)$. Furthermore when we substitute (7) into (2) we see that together with the finite contribution (i.e., the second term in (3)) the cross section becomes independent of the factorization scale $\mu_{F}$. This means that for the 
cancellation of the $\mu_{F}$ dependence only the LO FF is needed. Nonetheless, in order to see the influence of the NLO corrections to $D_{q \rightarrow \gamma}\left(z, \mu_{F}\right)$ we shall evaluate the $\gamma+(n+1)$-jet cross section also with the inclusion of the NLO photon FF.

Similarly to (7) the NLO FF $D_{q \rightarrow \gamma}\left(z, \mu_{F}\right)$ is obtained as the solution of (6), but now with $\mathrm{O}\left(\alpha \alpha_{s}\right)$ terms added on the right-hand side of (6). The result at scale $\mu_{F}$ is

$$
\begin{aligned}
& D_{q \rightarrow \gamma}\left(z, \mu_{F}\right)=\frac{\alpha e_{q}^{2}}{2 \pi}\left[P_{q \gamma}^{(0)}(z)+\frac{\alpha_{s}}{2 \pi} C_{F} P_{q \gamma}^{(1)}(z)\right] \ln \left(\frac{\mu_{F}^{2}}{\mu_{0}^{2}}\right) \\
& +\frac{\alpha_{s}}{2 \pi} C_{F} P_{q q}^{(0)}(z) \ln \left(\frac{\mu_{F}^{2}}{\mu_{0}^{2}}\right) \otimes\left[\frac{\alpha e_{q}^{2}}{2 \pi} \frac{1}{2} P_{q \gamma}^{(0)}(z) \ln \left(\frac{\mu_{F}^{2}}{\mu_{0}^{2}}\right)+D_{q \rightarrow \gamma}\left(z, \mu_{0}\right)\right]+D_{q \rightarrow \gamma}\left(z, \mu_{0}\right)
\end{aligned}
$$

where $P_{q \gamma}^{(1)}(z)$ is the next-to-leading order quark-to-photon splitting function [16] and $P_{q q}^{(0)}(z)$ is the well-known LO $q q$ splitting function. $D_{q \rightarrow \gamma}\left(z, \mu_{0}\right)$ is the initial value of the NLO FF, which contains all unknown long-distance contributions. The result in (9) is an exact solution of the evolution equation up to $\mathrm{O}\left(\alpha \alpha_{s}\right)$. Based on the above arguments, also the NLO photon FF has recently been determined [7] using the ALEPH $\gamma+1$-jet data [12]. A three parameter fit with $\alpha_{s}=0.124$ (this value for $\alpha_{s}$, just a scale-independent parameter here, was chosen so that the observed total $e^{+} e^{-}$annihilation cross section into hadrons is reproduced in the $\mathrm{O}\left(\alpha_{s}\right)$ calculation) yields [7]

$$
D_{q \rightarrow \gamma}^{N L O}\left(z, \mu_{0}\right)=\frac{\alpha e_{q}^{2}}{2 \pi}\left(-P_{q \gamma}^{(0)}(z) \ln (1-z)^{2}+20.8(1-z)-11.07\right)
$$

with $\mu_{0}=0.64 \mathrm{GeV}$. Inside the experimental errors this fit for the photon $\mathrm{FF}$ at $\mu_{0}$ describes the ALEPH data as good as the LO fit (8) [7, 9]. A fit with a larger value of $\alpha_{s}$ is also reported in [9].

It should be noted that the LO and NLO FF's of the photon as given in (7) together with (8) and in (9) together with (10) do not take into account resummation of powers of $\ln \left(\mu_{F}^{2} / \mu_{0}^{2}\right)$ as usually implemented, e.g. via Altarelli-Parisi evolution [17]. Such resummations are only unambiguous if the resummed logarithm is the only large logarithm in the kinematical region under consideration. If logarithms of different arguments can become simultaneously large, the resummation of one of these logarithms at a given order implies that all other potentially large logarithms are shifted into a higher order of the perturbative expansion, i.e. are neglected. In the evaluation of the $\gamma+1$-jet rate at $O(\alpha)$ 9] and $O\left(\alpha \alpha_{s}\right)$ 111 at LEP for $0.7<z<1$, one encounters at least two different potentially large logarithms, $\ln \mu_{F}^{2}$ and $\ln (1-z)$. In the high $z$ region, the region where the photon is isolated or almost isolated it is by far not clear that $\ln \mu_{F}^{2}$ is the largest logarithm. Choosing not to resum the logarithms of $\ln \mu_{F}$ is therefore equally justified for the case of large $z \rightarrow 1$.

In the conventional approach, the parton-to-photon FF's $D_{i \rightarrow \gamma}\left(z, \mu_{F}\right)$ satisfy a system of inhomogeneous evolution equations [17]. Usually these equations are diagonalized in terms of the singlet and non-singlet quark FF's as well as the gluon FF. For the case that the gluon-to-photon fragmentation is neglected, which we shall do in our application to 
DIS $\gamma+$ jets production, these equations can be simplified [8]. Then the flavor singlet and non-singlet quark-to-photon FF's become equal to a unique function $D_{q \rightarrow \gamma}$. This function satisfies the all-orders evolution equation

$$
\frac{d D_{q \rightarrow \gamma}\left(z, \mu_{F}\right)}{d \ln \mu_{F}^{2}}=\frac{\alpha e_{q}^{2}}{2 \pi} P_{q \gamma}^{(0)}+\frac{\alpha_{s}\left(\mu_{F}\right)}{2 \pi} P_{q q}^{(0)}(z) \otimes D_{q \rightarrow \gamma}\left(z, \mu_{F}\right)
$$

which has a similar form as the next-to-leading order evolution in the fixed-order approach, but the coupling $\alpha_{s}$ is not fixed and now is taken as a function of the factorization scale $\mu_{F}$. The full solution $D_{q \rightarrow \gamma}\left(z, \mu_{F}\right)$ of the all-orders inhomogeneous evolution equation is a sum of two contributions. The first term is the point-like (or perturbative) part $D_{q \rightarrow \gamma}^{p l}\left(z, \mu_{F}\right)$, which in the leading logarithmic (LL) approximation is a solution of the inhomogeneous equation (11). The second term is the hadronic (or non-perturbative) part $D_{q \rightarrow \gamma}^{h a d}\left(z, \mu_{F}\right)$, which is the solution of the corresponding homogeneous equation. This term must be fitted to experimental data to obtain the input at a starting scale. At LL only terms of the form $\alpha_{s}^{n} \ln ^{n+1} \mu_{F}^{2}$ are kept while beyond leading logarithms (BLL) both the leading and the sub-leading $\left(\alpha_{s}^{n} \ln ^{n} \mu_{F}^{2}\right)$ logarithms of $\mu_{F}$ are resummed to all orders in the strong coupling constant $\alpha_{s}$. Thus the LO solution (7) is the $n=0$ term of the LL approximation, whereas the NLO solution (9) is the BLL approximation up to the order $n=1$. By dropping the $\alpha_{s} \ln \mu_{F}^{2}$ terms in (9) one can also infer the LL approximation up to the order $n=1$.

In this conventional approach, the quark-to-photon fragmentation function is regarded as being of $O\left(\alpha / \alpha_{s}\right)$. In fact, the $n=0$ term of the LL approximation is proportional to $\ln \left(\mu_{F}^{2} / \mu_{0}^{2}\right)$. At large scales $\mu_{F}^{2} \gg \mu_{0}^{2}$ and assuming that $\mu_{F}$ can be identified with the scale that determines $\alpha_{s}$, i.e. $\alpha_{s} \sim 1 / \ln \mu_{F}^{2}$, the LL FF becomes of the order of $\mathrm{O}\left(\alpha / \alpha_{s}\right)$. This motivates the usual statement that the total fragmentation contribution is like $\mathrm{O}(\alpha)$, i.e. of the same order as the $\mathrm{LO}$ direct photon contribution. Of course, this statement depends on the considered kinematic region. With this argument however, one usually justifies the inclusion of terms in the fragmentation contribution of $\mathrm{O}\left(\alpha \alpha_{s}^{2}\right)$ which become of $\mathrm{O}\left(\alpha \alpha_{s}\right)$ if the $\mathrm{FF}$ is considered of $\mathrm{O}\left(\alpha / \alpha_{s}\right)$.

The most recent parametrizations of photon FF's in the conventional approach as described in the previous paragraph are those of GRV [5] and BFG [6]. The FF's of GRV [5] exist in the LL as well as in the BLL form, which in the following we shall denote as the LO and NLO FF's of GRV. However, the FF of BFG [6] are available only in the BLL approximation. They both have been compared to the ALEPH $\gamma+1$-jet cross section [12], which is sensitive to the large $z$ region $(0.7<z<1.0)$. The BFG prediction goes through the experimental points whereas the GRV NLO parametrization lies systematically higher and agrees less well with the ALEPH data [8, [3]. This difference can be attributed to both the choice of the input scale $\mu_{0}$ and of the non-perturbative input at this scale. The $\mathrm{BFG}$ input is smaller and according to the ALEPH data this choice is preferred.

Thus we have at least five different versions of photon FF's at our disposal which have been compared to the ALEPH data: two LO parametrizations, the one using the ALEPH data directly to determine the initial distribution given in (7) and (8) and the GRV 
parametrization in LO; and three NLO choices, the one written in (9) and (10) directly fitted to the ALEPH data, and the NLO parametrizations of GRV and BFG. We shall use these five parametrizations to predict the $\gamma+(n+1)$-jet cross section in deep-inelastic ep scattering.

\section{Numerical Results}

The results for these cross sections are obtained for energies and kinematical cuts appropriate for the HERA experiments. The energies of the incoming electron and proton are $E_{e}=27.5 \mathrm{GeV}$ and $E_{p}=820 \mathrm{GeV}$, respectively. The cuts on the DIS variables are as in our previous work [14]

$$
\begin{gathered}
Q^{2} \geq 10 \mathrm{GeV}^{2}, \quad W \geq 10 \mathrm{GeV}, \\
10^{-4} \leq x \leq 0.5, \quad 0.05 \leq y \leq 0.95 .
\end{gathered}
$$

To reduce the background from lepton radiation [15] we require

$$
90^{\circ} \leq \theta_{\gamma} \leq 173^{\circ}, \quad \theta_{\gamma e} \geq 10^{\circ}
$$

where $\theta_{\gamma}$ is the emission angle of the photon measured with respect to the momentum of the incoming electron in the HERA laboratory frame. The cut on $\theta_{\gamma e}$, the angle between the photon and the outgoing electron momentum, suppresses radiation from the final-state electron. The $\gamma$ and the hadron jet $J$ are required to have minimal transverse momenta

$$
p_{T, \gamma} \geq 5 \mathrm{GeV}, \quad p_{T, J} \geq 6 \mathrm{GeV} .
$$

Different values of minimal $p_{T}$ 's for the photon and the jet have to be chosen in order to avoid the otherwise present infrared sensitivity of the NLO predictions [14]. The PDF's of the proton are taken from MRST [18]. $\alpha_{s}$ is calculated from the two-loop formula with the same $\Lambda$ value $\left(\Lambda_{\overline{M S}}\left(n_{f}=4\right)=300 \mathrm{MeV}\right)$ as used in the MRST parametrization. The scale in $\alpha_{s}$ and the factorization scales $\mu_{F}$ for the proton PDF and the photon FF are equal and fixed to $\sqrt{Q^{2}}$.

We are interested in the differential cross section $d \sigma / d z_{\gamma}$ at NLO (up to $\mathrm{O}\left(\alpha \alpha_{s}\right)$ ) as a function of $z_{\gamma}$, where

$$
z_{\gamma}=\frac{p_{T, \gamma}}{p_{T, \gamma}+p_{T, h a d}} .
$$

This definition of $z_{\gamma}$ agrees with the one in (5) for the fragmentation contribution. In (15), $p_{T, h a d}$ is the transverse momentum of the parton producing hadrons, which is recombined with the photon into the photon jet. Here the photon is treated like any other parton during recombination, so that one of the recombined jets may be the photon jet. For the recombination of the two partons into a hadron jet or a parton and the photon into the photon jet, we use the cone algorithm of the Snowmass convention [19]. The recombination 


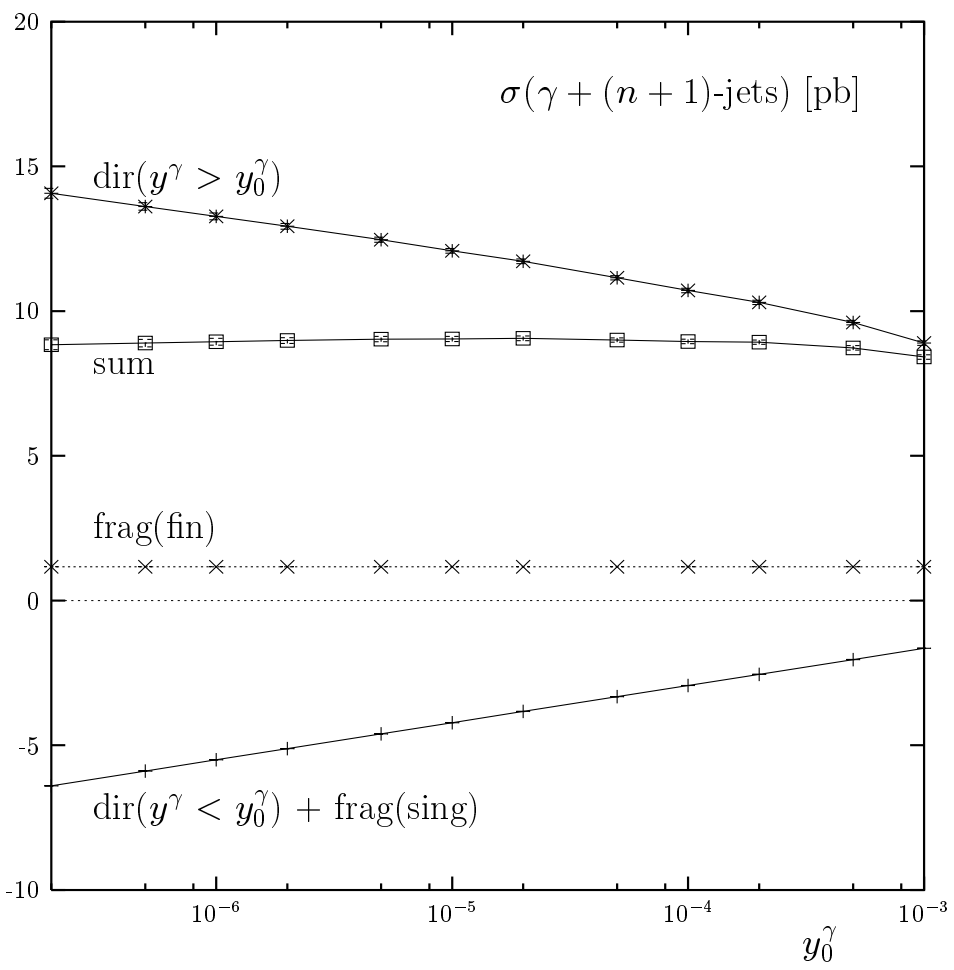

Figure 1: $y_{0}^{\gamma}$-dependence of separate contributions to the $\gamma+(n+1)$-jets cross section.

is applied in the $\gamma+2$ parton sample and yields a contribution to the $\gamma+(1+1)$-jet class. The $\gamma+(2+1)$-jet class consists of the unrecombined contributions of the $\gamma+2$ parton sample. The recombination is performed in the $\gamma^{*} p$ center-of-mass frame. Two partons $i$ and $j$ are combined into a jet $J$ if they obey the cone constrains $R_{i, J}<R$ and $R_{j, J}<R$, where

$$
R_{i, J}=\sqrt{\left(\eta_{i}-\eta_{J}\right)^{2}+\left(\phi_{i}-\phi_{J}\right)^{2}}
$$

$\eta_{J}$ and $\phi_{J}$ are the rapidity and the azimuthal angle of the recombined jet. If for example, parton $i$ is the $\gamma$, then $J$ is the photon jet. The jet variables $\eta_{J}$ and $\phi_{J}$ are obtained from the averages of the corresponding variables of the recombined partons $i$ and $j$ multiplied with their respective $p_{T}$ values and $p_{T, J}=p_{T, i}+p_{T, j}$. We choose $R=1$. The azimuthal angle is defined with respect to the scattering plane defined by the momenta of the ingoing and outgoing electron. It is known that the cone algorithm is ambiguous for final states with more than three particles or partons. Since we have maximally three partons in the final state this is not relevant in our case. Furthermore it will be no problem to repeat the calculation for any other cluster algorithm that might be used in the analysis of forthcoming experimental data. It is obvious that for the fragmentation contribution the cone constraint is always satisfied since the hadronic remnant in the fragmentation is collinear with the photon.

Before we present our results for $d \sigma / d z_{\gamma}$ for the various choices of the photon FF we 


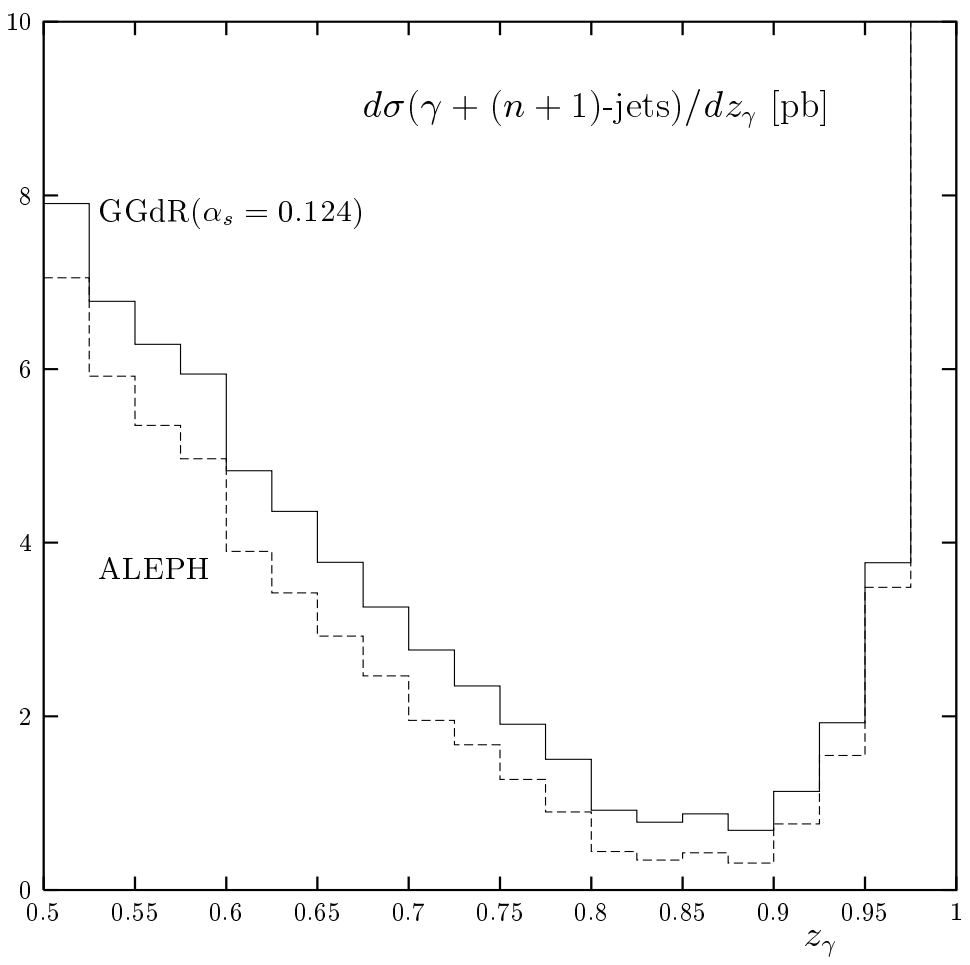

Figure 2: Comparison of the $\gamma+(n+1)$-jet cross section for the LO and NLO parametrizations of the FF obtained from fits to the ALEPH data.

show that the cross section is independent of the slicing cut $y_{0}^{\gamma}$. For this purpose we have calculated the sum of the cross sections for the production of $\gamma+(n+1)$-jets $(n=1,2)$ as a function of $y_{0}^{\gamma}$ in the range $2 \cdot 10^{-7}<y_{0}^{\gamma}<10^{-3}$. The result is shown in Fig. 目. Here we have plotted the cross section for the direct contribution with $y_{\gamma}>y_{0}^{\gamma}$ which increases with decreasing $y_{0}^{\gamma}$ and the direct contribution for $y_{\gamma}<y_{0}^{\gamma}$ together with the second term in contribution (3) inserted in (2) which we denote by 'frag(sing)' in the figure. This latter contribution is negative and decreases with decreasing $y_{0}^{\gamma}$ in such a way that the sum of the increasing and decreasing contributions is constant inside the numerical accuracy for $y_{0}^{\gamma}<5 \cdot 10^{-4}$. The sum shown in Fig. [1 contains also the fragmentation contribution (2) with the photon FF $D_{q \rightarrow \gamma}\left(z, \mu_{F}^{2}\right)$ as given in (7) and (8) (denoted by 'frag(fin)' in the figure). Of course, this contribution is independent of $y_{0}^{\gamma}$. It amounts to approximately $14 \%$ of the total $\gamma+(n+1)$-jet cross section. The cross sections shown in Fig. 1 are calculated with the kinematical cuts specified above and the additional cut $z_{\gamma} \geq 0.5$. From Fig. [1 we conclude that the slicing cut for the photon should be chosen smaller than $10^{-4}$ to obtain a reliable cross section. For the following results we fixed it to $y_{0}^{\gamma}=10^{-5}$ as in our previous work [14].

The results for $d \sigma / d z_{\gamma}$ as a function of $z_{\gamma}$ are shown in Figs. 2, 3 and 1 . The total cross section is dominated by the last bin $0.975<z_{\gamma} \leq 1$ which is clipped off above $10 \mathrm{pb}$ in these figures. In this bin we have the direct contribution and those fragmentation 
contributions with no or little hadronic remnant. The fraction of the total cross section (for $0.5 \leq z_{\gamma} \leq 1$ ) contained in the last bin amounts to $70 \%$ to $80 \%$ and does not depend on the parametrization of the FF. Therefore it is clear that the dependence of the photon plus jet cross section on $z_{\gamma}$ below $z_{\gamma}=1$ is an appropriate observable which contains information on the photon FF. Here the fragmentation contribution dominates and in addition we have those direct contributions from the $(\gamma+2)$-parton sample where the photon is recombined with one of the partons. In Fig. 2 the cross section $d \sigma / d z_{\gamma}$ in the region $0.5<z_{\gamma}<1.0$ is plotted for the photon FF's from (7) and (8) (denoted ALEPH) and from (9) and (10) (denoted GGdR $\left(\alpha_{s}=0.124\right)$ in the figure). For both cases the cross section first decreases with increasing $z_{\gamma}$ until it reaches a minimum near $z_{\gamma}=0.9$, from which it increases strongly towards $z_{\gamma}=1$. This dependence is similar to what has been found for the photon plus one-jet cross section in $e^{+} e^{-}$annihilation at the $Z$-resonance [7, 9]. Note however, that below $z_{\gamma}=0.5$, i.e. outside the range shown in these figures, the cross section decreases again towards smaller values of $z_{\gamma}$. Unlike at LEP, the photon-jet is here required to have a minimal transverse momentum as given by (15). The decrease in the cross section for values of $z_{\gamma}$ below 0.5 is a kinematical effect resulting from the imposition of this cut.

For the two choices of FF's the predicted cross section differs by approximately $20 \%$. The cross section for the LO FF is smaller than for the NLO FF. We note that the $\alpha_{s}$ value quoted for this $\mathrm{FF}$ is not the value for which the cross section was calculated; rather it is the value which was chosen to fit the photon FF to the ALEPH data. Both photon FF's fit the ALEPH data in LO and NLO approximation. In our case only the LO $\mathrm{FF}$ is appropriate. The result for the NLO FF gives just an indication of higher-order corrections up to $\mathrm{O}\left(\alpha \alpha_{s}^{2}\right)$. However, it is not a full prediction at this order since the NNLO calculation for the direct contribution has not been done, the NLO corrections to $|M|^{2}$ on the right-hand-side of (2) are not included either.

In Fig. 3 the predictions with GRV [5] and BFG [6] FF's are shown. Both are FF's in NLO and with all leading and subleading logarithms of $\ln \left(\mu_{F}^{2} / m_{0}^{2}\right)$ resummed at the nextto-leading logarithmic accuracy. The behavior of the cross section as a function of $z_{\gamma}$ is qualitatively similar to the result in Fig. 2. The cross section for GRV is larger than the one with the BFG FF. This is to be expected since GRV gives a larger $e^{+} e^{-}$cross section for $(\gamma+1)$-jet than BFG also for the ALEPH kinematical conditions.

Comparing with the fixed-order result in Fig. 2 the BFG cross section lies above the fixedorder prediction obtained using the LO FF over the whole $z_{\gamma}$ region. The discrepancy is largest in the region $0.65<z_{\gamma}<0.95$ where the cross section is smaller. At the minimum the two cross sections differ by more than a factor two.

In Fig. 1 the results for the GRV photon FF's are displayed, but now for both the LO and the NLO parametrization. On the average the cross section for the LO fragmentation function is again smaller by $20 \%$ compared to the NLO prediction. In addition, we have plotted the cross sections for the point-like approximations of the FF's. This approximation is rather good, i.e. the influence of the hadronic part of the FF is small in the 


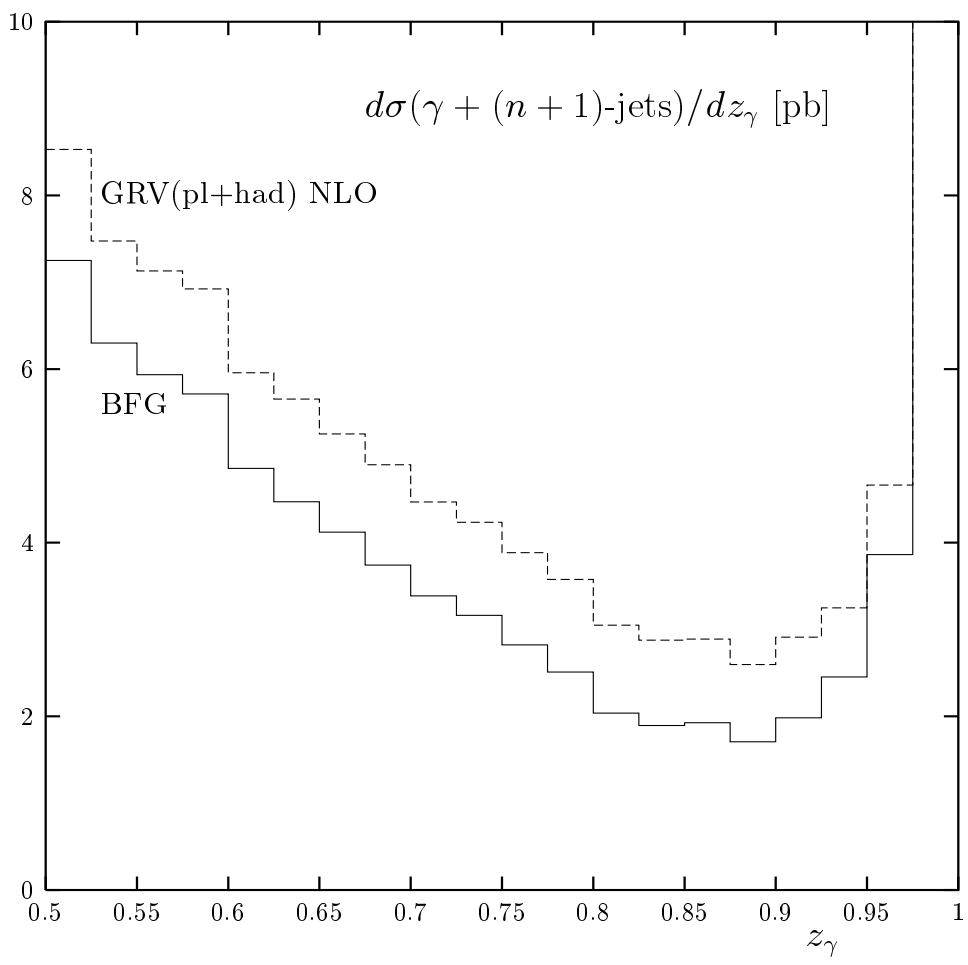

Figure 3: Comparison of the $\gamma+(n+1)$-jet cross section for the NLO parametrizations of GRV and BFG.

considered $z_{\gamma}$ range. We note that the difference between the full FF and the point-like part increases with decreasing $z_{\gamma}$.

If we compare our results for the various photon FF's in Figs. 2, 3 and 4 we observe that the predictions agree approximately within $20 \%$ in the small and large $z_{\gamma}$ regions, i.e. for $z_{\gamma}<0.65$ and $z_{\gamma}>0.90$. However, near the minimum of the cross section, i.e. in the region $0.65<z_{\gamma}<0.95$, the results differ by up to a factor two. The largest differences occur between the predictions obtained with the ALEPH photon fragmentation function on the one side and the GRV and BFG parametrizations on the other side. This difference comes mainly from the fact that different evolution approaches are used. Whereas for GRV and $\mathrm{BFG}$ the $\mathrm{FF}$ at $\mu_{F}^{2}=Q^{2}$ is obtained from the conventional evolution after the leading and/or subleading logarithms of $\mu_{F}$ were resummed, the ALEPH FF's are evolved only to the respective finite order in $\alpha_{s}$ as given in (7) and (9). Therefore, if we calculated $d \sigma / d z_{\gamma}$ for $e p \rightarrow e \gamma+(n+1)$-jets at the large scale $\mu_{F}=M_{Z}$ the cross sections obtained for BFG and ALEPH would come out quite similar over the whole $z_{\gamma}$ range inside the $20 \%$ margin. Only when we go to the scale $\mu_{F}^{2}=Q^{2}$, which on the average is much smaller, we observe that the cross section obtained using the BFG photon fragmentation function is much larger than the ALEPH cross section in the region $0.65<z_{\gamma}<0.95$.

Provided the resummed solution of the all-orders evolution equation can be accurately 


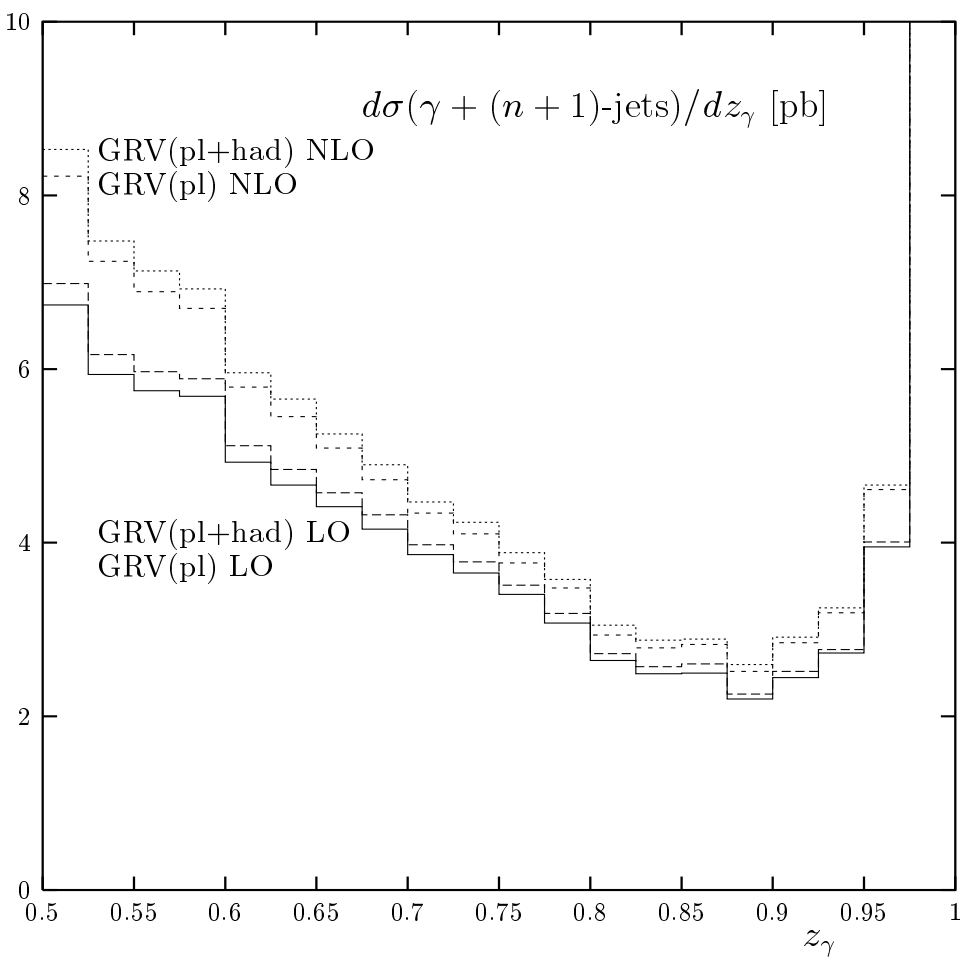

Figure 4: Comparison of the $\gamma+(n+1)$-jet cross section for LO and NLO parametrizations of GRV.

determined [8] over the whole $z_{\gamma}$ range under consideration, i.e. for $0.5<z_{\gamma}<1$, the approach using this solution represents the theoretically preferred approach as it is the most complete. The approach using an expanded and therefore approximated photon FF has however important advantages. As already mentioned, its use leads to factorization scale independent results for the cross section evaluated at a given fixed order in $\alpha_{s}$. Moreover it enables an analytic determination of the photon FF.

Within the conventional approaches, the BFG prediction should be preferred over the result with the GRV FF. The difference between GRV and BFG in Fig. 3 is related to the choice of a different input at the starting scale $\mu_{0}$. As already mentioned, the BFG parametrization is in better agreement with the ALEPH data [8] than the GRV NLO parametrization and therefore is the more realistic choice of $\mathrm{FF}$ for the photon. This could be further tested as soon as data from HERA on photon plus jet production in DIS become available. 


\section{Conclusions}

We have presented a NLO calculation for the production of photons accompanied by jets in deep inelastic electron proton scattering, taking into account the contribution from quarkto-photon fragmentation. We have calculated the cross section as a function of $z_{\gamma}$ for those parametrizations of the photon fragmentation functions, which had been compared to photon plus jet data from the ALEPH collaboration. As we observed significant differences between the predictions, we expect that the measurement of photon plus jet production in DIS at HERA will contribute to testing these photon fragmentation functions.

\section{Acknowledgements}

A. G. would like to thank A. Wagner for financial support during her stay at DESY where part of this work has been carried out.

\section{References}

[1] K. Koller, T. F. Walsh and P. M. Zerwas, Z. Phys. C2 (1979) 197; E. Laermann, T. F. Walsh. I. Schmitt and P. M. Zerwas, Nucl. Phys. B207 (1982) 205

[2] S. Frixione, Phys. Lett. B429 (1998) 369

[3] K. Ackerstaff et al., OPAL Collaboration, Eur. Phys. J. C2 (1998) 39

[4] D. W. Duke and J. F. Owens, Phys. Rev. D26 (1982) 1600; J. F. Owens, Rev. of Mod. Phys. 59 (1987) 465

[5] M. Glück, E. Reya and A. Vogt, Phys. Rev. D48 (1993) 116

[6] L. Bourhis, M. Fontannaz and J. Ph. Guillet, Eur. Phys. J. C2 (1998) 529

[7] A. Gehrmann-De Ridder, T. Gehrmann and E. W. N. Glover, Phys. Lett. B414 (1997) 354

[8] A. Gehrmann-De Ridder and E. W. N. Glover, Eur. Phys. J. C7 (1999) 29

[9] A. Gehrmann-De Ridder and E. W. N. Glover, Nucl. Phys. B517 (1998) 269

[10] Z. Kunszt and Z. Trócsány, Nucl. Phys. B394 (1993) 139

[11] E. W. N. Glover and A. G. Morgan, Z. Phys. C62 (1994) 311

[12] D. Buskulic et al., ALEPH Collaboration, Z. Phys. C69 (1996) 365 
[13] A. Gehrmann-De Ridder, Proc. Workshop on Photon Interactions and the Photon Structure, Lund, Sweden, 10-13 Sep 1998, Eds. G. Jarlskog and T. Sjöstrand, p. 147 (hep-ph/9810507)

[14] A. Gehrmann-De Ridder, G. Kramer and H. Spiesberger, DESY 99-035, MZ-TH/9905,TTP 99-13, hep-ph/9903377, Phys. Lett. B (in press)

[15] G. Kramer, D. Michelsen and H. Spiesberger, Eur. Phys. J. C5 (1998) 293

[16] G. Curci, W. Furmanski and R. Petronzio, Nucl. Phys. B175 (1980) 27; W. Furmanski and R. Petronzio, Phys. Lett. 97B (1980) 437

[17] G. Altarelli and G. Parisi, Nucl. Phys. B126 (1977) 298

[18] A. D. Martin, R. G. Roberts, W. J. Stirling and R. S. Thorne, Eur. Phys. J. C4 (1998) 463

[19] J. E. Huth et al., Proc. Summer Study on High Energy Physics, Research Directions for the Decade, Snowmass, 1990; F. Abe et al., CDF Collaboration, Phys. Rev. D45 (1992) 1448; M. Seymour, Z. Phys. C62 (1994) 127 